\title{
Dilated cardiomyopathy and functional mitral regurgitation complicated with traumatic ventricular septal defect
}

Hisato Ito, MD, Kiyohito Yamamoto, MD, and Takane Hiraiwa, MD, Shizuoka, Japan

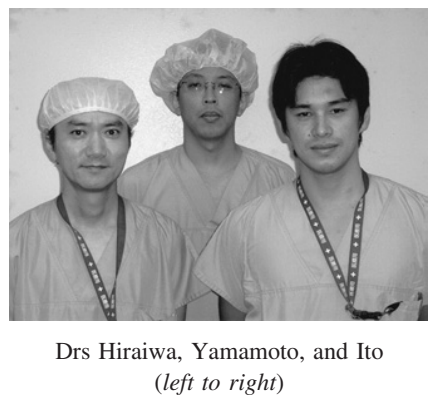

W e report a case of dilated cardiomyopathy (DCM) and functional mitral regurgitation (MR) complicated with a traumatic ventricular septal defect (VSD), which was managed by means of intracardiac left ventricular restoration (LVR) ${ }^{1}$ and VSD closure 40 years after blunt chest trauma.

\section{Clinical Summary}

A 66-year-old man presented with a clinical finding of severe heart failure. He had sustained blunt chest and abdominal trauma in a motor vehicle collision, followed by an emergency laparotomy, at the age of 26 years. He had been in good health until he was 49 years old, when he had recurrent episodes of palpitation and chest discomfort. Precordial examination disclosed a 3/6 holosystolic murmur, which had not been heard before. Left ventriculographic analysis revealed a muscular VSD and akinetic anteroseptal wall.

From the Division of Cardiovascular Surgery, Hamamatsu Medical Center, Shizuoka, Japan.

Received for publication July 31, 2007; accepted for publication Aug 7, 2007.

Address for reprints: Hisato Ito, MD, Division of Cardiovascular Surgery, Hamamatsu Medical Center, 328 Nakaku Tomitsuka, Hamamatsu, Shizuoka 432-8580, Japan (E-mail: hisato.ito@gmail.com).

J Thorac Cardiovasc Surg 2007;134:1583-4

$0022-5223 / \$ 32.00$

Copyright () 2007 by The American Association for Thoracic Surgery doi:10.1016/j.jtcvs.2007.08.014
He was medically treated because the left-to-right shunt was relatively small, with a 1.39 pulmonary/systemic flow ratio $(\mathrm{Qp} /$ Qs). His clinical condition remained satisfactory until the age of 59 years, when he started to complain of dyspnea on exertion. Transthoracic echocardiographic analysis revealed a dilated left ventricle with diffuse hypokinetic wall, severe MR, and the VSD (Figure 1). The left ventricular ejection fraction was $30.5 \%$. The left ventricular end-diastolic, left ventricular end-systolic, and left atrial diameters were $68.5,58.3$, and $45.4 \mathrm{~mm}$, respectively. Although he was taking medication with diuretics and digoxin, he repeatedly had heart failure and had the worst symptom at the age of 66 years. The Qp/Qs was 1.10, and there was no coronary artery disease on repeat cardiac catheterization. He underwent surgical intervention after 7 months of rigorous medical control of the heart failure. The New York Heart Association functional class had improved from IV to II before the operation. Intracardiac LVR of papillary muscle sling, concomitant mitral annuloplasty (MAP) and tricuspid annuloplasty, and patch closure of the VSD was performed (Figure 2). The left ventricle was opened from the apex to the middle part, with an incision parallel to the left anterior descending artery. There was a scar lesion over the ventricular septum, at the center of which was 5-mm VSD. There were no scar lesions in any other part of the myocardium. The abnormal displacement of the papillary muscles was corrected with a 5-mm Gore-Tex tube (WL Gore, Flagstaff, Ariz) encircling the base of the papillary muscles, which banded them side-by-side. There were no mitral leaflet abnormalities. The mitral valve repair was completed by means of MAP with an undersized annuloplasty ring (26-mm Physio-ring; Edwards
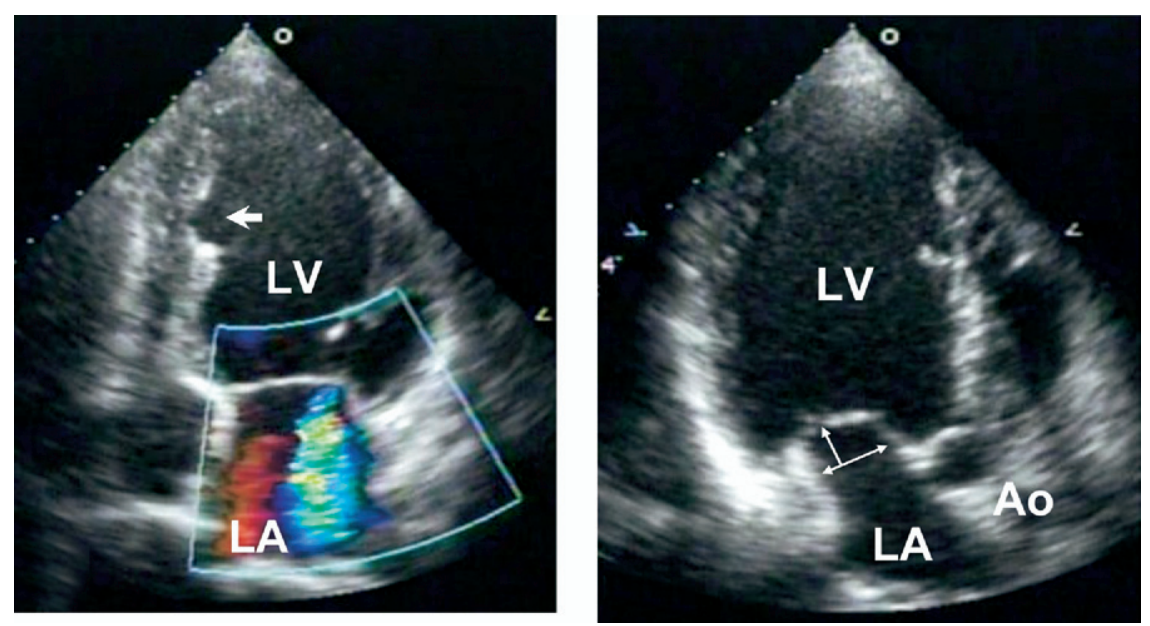

Figure 1. Preoperative transthoracic echocardiographic scan showing ventricular septal defect (arrow) and severe mitral regurgitation (left panel) and dilated left ventricle and tethering of the mitral leaflets (right panel). $L V$, Left ventricle; $L A$, left atrium; Ao, aorta. 

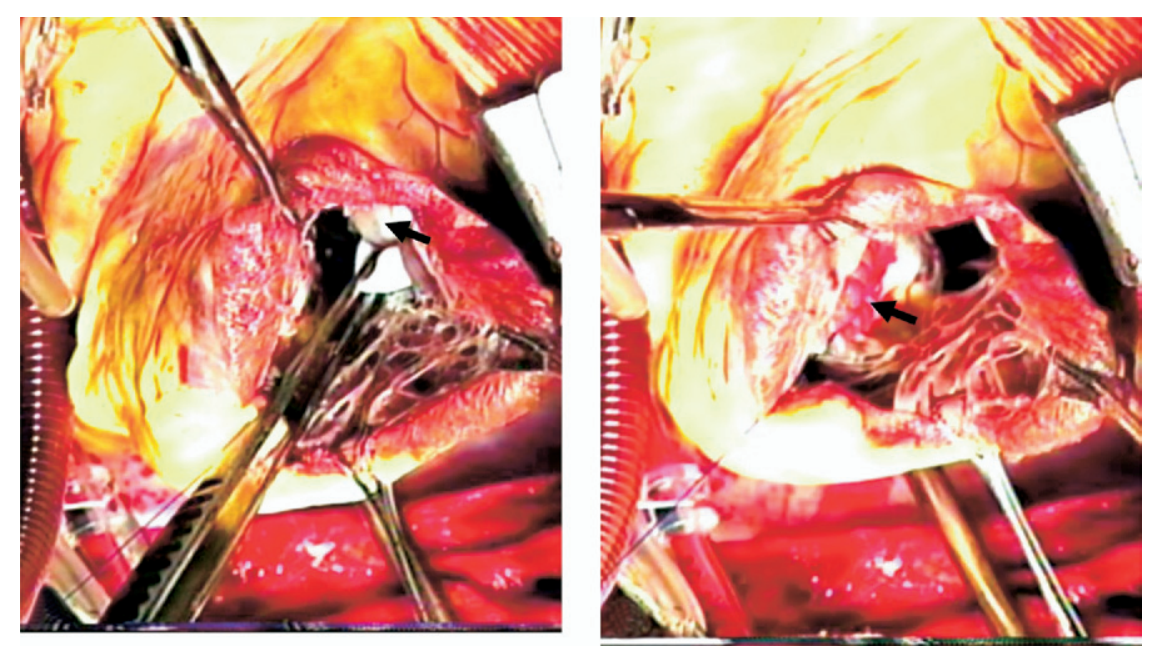

Figure 2. Intraoperative photographs showing part of the papillary muscle sling (forceps) and the posterior papillary muscle (arrow, left panel) and showing the closed VSD with a patch (arrow) around which a scar area was present (right panel).

Lifesciences, Irvine, Calif). Tricuspid annuloplasty was performed with the DeVega technique. The postoperative course was favorable. Transthoracic echocardiographic analysis at discharge showed neither persistent left-to-right shunt nor valvular regurgitation. The left ventricular ejection fraction, left ventricular end-diastolic diameter, left ventricular end-systolic diameter, and left atrial diameter were $36.1 \%$ and $53.5,45.4$, and $35.1 \mathrm{~mm}$, respectively.

\section{Discussion}

Traumatic VSD occurring immediately after blunt chest trauma is caused by acute compression of the heart. In contrast, its delayed presentation results from septal myocardial infarction. ${ }^{2}$ In the present case, the cause of the VSD was likely to have been posttraumatic myocardial infarction, taking into consideration the fact that the murmur was first noticed many years after the traumatic event and that there was a scar lesion over the ventricular septum. A conservative approach is recommended for small traumatic VSD with a pulmonary-to-systemic shunt of less than $2: 1,^{3}$ and this VSD per se would not have required surgical repair. However, the decision to perform surgical repair was made because of the presence of heart failure, which resulted from ventricular dilatation and diminished cardiac function. The Qp/Qs was too small to cause volume overload and ventricular dilatation. Considering the fact that the anteroseptal wall had initially been impaired and then depression of the global wall motion had progressed over the years, it was likely that idiopathic DCM developed in his middle age, and the vicious cycle of ventricular dilatation and functional MR played a key role in hemodynamic deterioration. If the global heart damage had been caused by myocardial contusion, diffusely impaired wall motion would have been observed at the moment of the trauma, and there would have been patchy scarring in the entire myocardium. ${ }^{4}$ In regard to controlling MR, the submitral approach was essential in addition to MAP, and papillary muscle sling was performed, which shortens the distance between the two papillary muscles and is considered an intracardiac method for LVR. ${ }^{1}$ In conclusion, we experienced a peculiar complex of the traumatic VSD and DCM with functional MR, which has not been reported before.

\section{References}

1. Hvass U, Tapia M, Baron F, Pouzet B, Shafy A. Papillary muscle sling: a new functional approach to mitral repair in patients with ischemic left ventricular dysfunction and functional mitral regurgitation. Ann Thorac Surg. 2003;75:809-11.

2. Genoni M, Jenni R, Turina M. Traumatic ventricular septal defect. Heart. 1997;78:316-8.

3. Perzada F, McDowell J, Cohen E. Traumatic ventricular septal defect: sequential hemodynamic observations. N Engl J Med. 1974 291:892-5.

4. Doty DB, Anderson AE, Rose EF, Go RT, Chiu CL, Ehrenhaft JL. Cardiac trauma: clinical and experimental correlations of myocardial contusion. Ann Surg. 1974;180:452-60. 